\title{
Systematic Review of Case Reports about Korean Medicine for Lung Cancer
}

\author{
Gajin Han $^{1,2^{*}}$, Haegue Shin ${ }^{1}$, Sin Seong ${ }^{1}$, Sungsu Kim ${ }^{1}$ \\ ${ }^{1}$ Soram Korean Medicine Hospital \\ ${ }^{2}$ Soram Korean Medicine Research Institute
}

Objectives: This systematic review aimed to put the case reports of lung cancer on Korean medicine (KM) together and adopt the results in clinical practice.

Methods: Researches were searched using the PubMed, EMBASE, OASIS, KoreanTK, KISTI, RISS, KISS, and NDSL. The search term were 'lung cancer' and KM. There was no restriction in year.

Results:

1. Among the 48 studies, 68 patients were reported in total. The types of lung cancer were non-small-cell lung cancer $(\mathrm{n}=41)$ and small-cell lung cancer $(\mathrm{n}=6)$.

2. The number of patients who received KM therapy alone was 40 . On the other hand, 25 patients were treated with KM and chemotherapy simultaneously. All case reports used herbal medicine except 2 studies. Other types of treatment were acupuncture, moxibustion, pharmacopuncture, cupping, meditation, etc.

3. Several efficacy evaluation variables were used such as tumor size, changes of symptoms, duration of survival, the quality of life, and so on. The safety was evaluated by checking adverse effects using blood test.

4. Regarding the tumor response, partial response was reported in 12 cases, stable disease was in 22 cases, $50 \%$ of the total cases, which is a high level of tumor response. Furthermore, all 11 cases with the evaluation on the length of survival showed prolonged survival than the expectancy of corresponding stage, with the stable quality of life.

Conclusion: We have found that the applicability of KM for treatment of lung cancer through this review. Evidence based medicine can be realized by checking cases and applying them in clinical practice.

\section{Key Words : Lung neoplasm, Medicine, Korean Traditional, Case reports, Systematic Review}

\section{Introduction}

Lung cancer is the major cause of deaths from cancer worldwide, and one of the common types of cancer. ${ }^{1)}$ According to the statistics of year 2017 in the United States, lung cancer accounts for $27 \%$ and $25 \%$ of deaths from cancer in men and women population respectively; ${ }^{2)}$ the recent research in Korea reported lung cancer is the second most prevalent cancer among men, the fifth among women, and the fourth in total. The five-year survival rate being $26.7 \%$ - the lowest among the top ten cancers, it is one of the fatal types of cancer. ${ }^{3)}$

The standard treatment of lung cancer is surgery, radiotherapy, and chemotherapy. The recurrence rate of lung cancer after surgery is relatively high, ${ }^{4}$ and more than $50 \%$ of lung cancer patients are found with metastasis at the time of diagnosis. This leads to

\footnotetext{
- Received : 7 December 2018

- Revised : 18 December 2018

- Accepted : 18 December 2018

- Correspondence to : Gajin Han

Soram Korean Medicine Research Institute, Seoul, Republic of Korea

Tel : +82-70-4803-6648, Fax : +82-70-8282-7001, E-mail : kmdhgj@soram.kr
} 
chemotherapy for most lung cancer patients, inducing side effects such as vomiting, diarrhea, loss of appetite, dyspnea, myelosuppression, and renal toxicity, degrading the quality of life. ${ }^{5)}$ However, the existing medical system is not sufficiently dealing with these symptoms and lacks intensive management. ${ }^{6}$ On the other hand, there are various approaches to overcome the limitation of existing treatment and to develop new therapeutic approaches in the academic society of Korean medicine, with a number of publications about the effect of them. ${ }^{\text {) }}$

The clinical case report is a type of medical writing on symptoms, diagnosis, progress, and treatment of one or several patients, originated from ancient Egypt. ${ }^{8)}$ Its value has been controversial for decades, ${ }^{9)}$ and is reckoned as the lowest grade in evidence based medicine, the movement arisen in the late 1980s. ${ }^{10)}$ Despite such controversies, the case report is important as a medical observation on the progress, ${ }^{11)}$ and promotes communication about discovery of new illness, mechanism of disease, therapeutic approach, and side effect and efficacy of drug among physicians. Furthermore, it has a potential of developing into new hypothesis for well-designed studies like randomized clinical trials. ${ }^{12)}{ }^{13)}$ It is also educational, helping readers acquire the new clinical knowledge and apply in clinical practice. ${ }^{14)}$

Recently, various case reports on lung cancer treated with Korean medicine (KM) or combined Korean and western medicine has been published by KM doctors, but so far there is no review on these studies to help have an insight to apply them in clinical practice. Thus, the author analyzed the case reports related to lung cancer until lately, and studied on the common treatments in use and their effect on prognosis to support clinical practice and to suggest the direction of further study.

\section{Method}

\section{Selection of study object}

We did not limit the period of time of publication, and the language was limited to Korean and English. The databases used for international research were PubMed and EMBASE and the databases for national studies were Oriental Medicine Advanced Searching Integrated System (OASIS, oasis.kiom.re.kr), Korean Traditional Knowledge Portal (KoreanTK, http://www.koreantk.com), Korea Institute of Science and Technology Information (KISTI, society.kisti.re.kr), Research Information Sharing Service (RISS, http://www.riss.kr), Koreanstudies Information Service System (KISS, kiss.kstudy.com), and National Digital Science Library (NDSL, www.ndsl.kr). The search term used on PubMed was "Lung Neoplasms"[MeSH Terms] AND "Medicine, Korean Traditional"'[MeSH Terms] and the one for EMBASE was 'lung tumor'/exp AND ('herbal medicine'/exp OR 'korean medicine'/exp) AND 'case report'/exp. In the national databases, the combination of "lung cancer (폐암)" and "Korean medicine (한의학)" were used.

\section{Analysis of selected literature}

Participants/Problems (P) included the type and the stage of lung cancer, Intervention (I) included the treatment patients received, and Outcome $(\mathrm{O})$ included which measurement was used to evaluate the effect and also included other characteristics.

\section{Result}

\section{Search result}

In the international database, two results from PubMed and 29 from EMBASE were searched. In Korean database, OASIS had 120 results from searching "lung cancer (폐암)" and 58 results in KoreanTK. As 


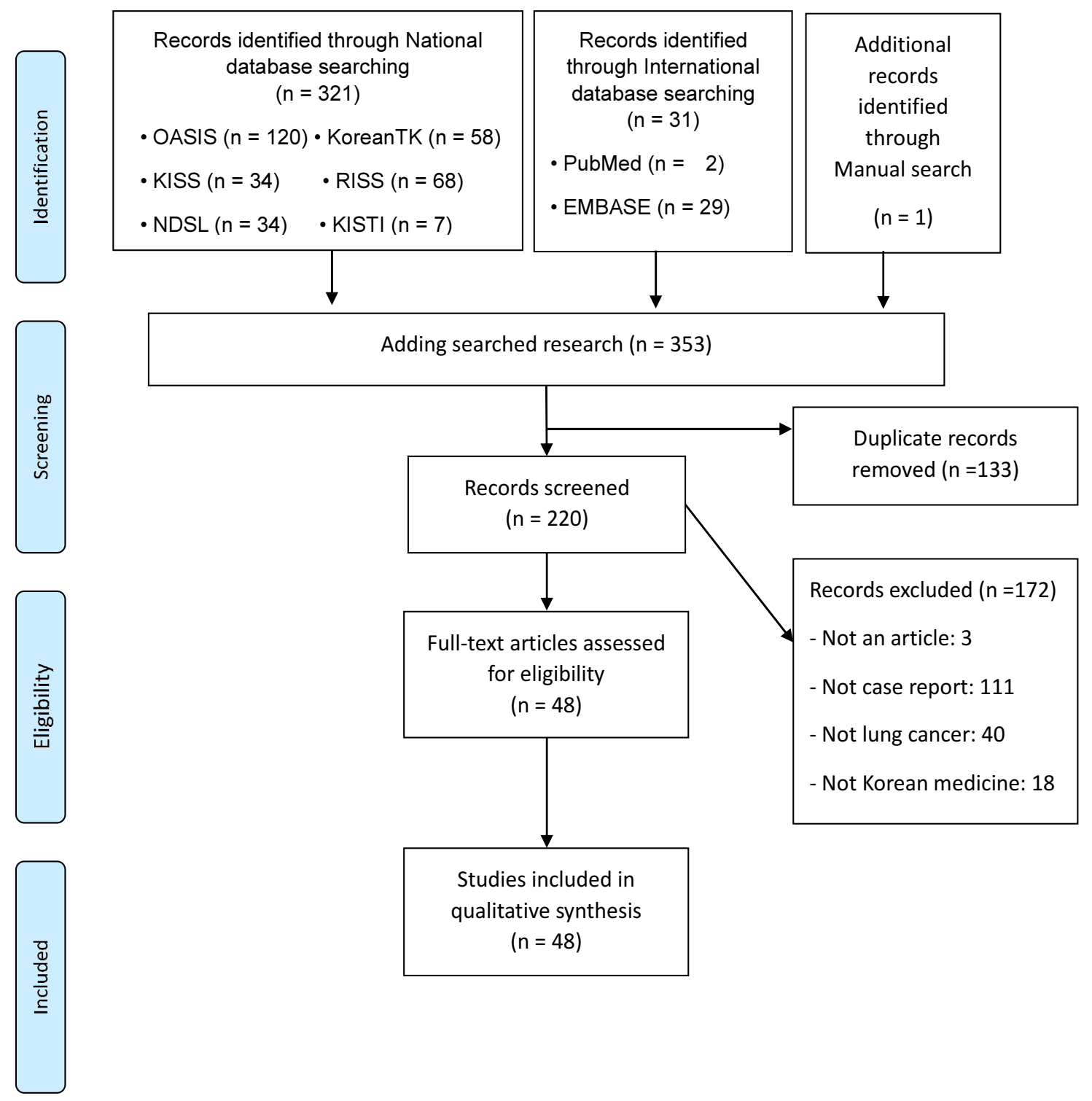

Fig. 1. Flow chart of study selection.

the result of searching "lung cancer (폐암) AND Korean medicine (한의학)," 34 studies were found in KISS, 68 in RISS, 34 in NDSL, and 7 in KISTI. One additional record was identified through manual research. All 353 studies were added together, and 133 duplicate records were removed. 220 studies were screened and
172 were excluded because they were not an article; a case report; about lung cancer; nor about Korean medicine. As an outcome, 48 full-text articles were assessed for eligibility and included in qualitative synthesis. (Figure 1) 


\section{Literature analysis}

\section{1) The number of studies by year}

There were 1 study in 1997 and 1999, 2 studies in 2003, 2005, 2007, 2008, 2009 and 3 in 2010, 7 in 2011, 3 in 2012, 1 in 2013, 6 in 2014, 5 in 2015, 2 in 2016, 3 in 2017, and 2 in 2018. From 1997 to this day, case reports on lung cancer has been steadily published, and there were many studies especially in 2011.

\section{2) The number of studies by journal}

Seven case reports were published in the international journal such as Integrative Cancer Therapies, Explore, Oncology Letters, and Case Reports in Oncology. Regarding domestic journals, 17 studies were published in the Journal of Korean Traditional Oncology, 3 studies in the Journal of pharmacopuncture, 1 in the Journal of Korean Medicine, 11 in the Journal of Internal Korean Medicine, 2 in the Journal of Oriental Chronic Diseases, 2 in the Journal of Korean Oriental Oncology, 2 in the Journal of physiology and Pathology in Korean Medicine, and 2 in the Journal of Sasang Constitutional Medicine.

\section{3) The subject of studies (the type of lung cancer)}

Among the 48 studies, 68 patients were reported in total. Except for 5 cases of cancer metastasized to lung ( 3 cases of primary hepatocellular cancer, 1 case of primary rectal cancer, 1 case of bladder cancer), other studies were primary lung cancer.

The types of lung cancer were non-small-cell lung cancer (NSCLC, $\mathrm{n}=41$ ) and small-cell lung cancer (SCLC, $\mathrm{n}=6$ ), with 8 unknown cases (undescribed in 7 cases and biopsy refused in one case). Among NSCLC studies, squamous cell carcinoma was reported in 12 cases, adenocarcinoma in 22 cases, and unknown in
7 cases; SCLC studies included 2 cases of limited stage,

1 case of extensive stage, and 3 unknown cases.

The number of lung cancer patients with a single metastasis were 33 - the sites were the opposite lung $(n=7)$, brain $(n=7)$, lymph nodes $(n=9)$, bone $(n=4)$, pleura $(n=6)$ and unknown in 2 cases. Multiple metastasis was reported in 7 cases: 1 patient to lymph nodes, bones, and chest wall; 1 patient to the brain and bones; 1 patient to the brain and the opposite lung; 2 patients to lymph nodes and the opposite lung; 1 patient to the lymph nodes, the opposite lung, and the body of pancreas. One patient was diagnosed as Mx, so it was described just as mediastinum and pericardial invasion. (Table 1)

\section{4) The types of treatment}

The number of patients who received KM therapy alone was 38. The reasons of taking KM therapy alone are to prevent recurrence/metastasis of tumor or to treat side effect after having finished standard western treatment such as surgery, chemotherapy, and radiotherapy; the refusal of patients to receive standard western treatment; and old age.

On the other hand, 25 patients were treated with KM and chemotherapy simultaneously. The chemotherapy drugs were olmutinib, crizotinib, gefitinib, gemcitabine, cisplatin, pemetrexed, erlotinib, bevacizumab, afatinib, paclitaxel, and carboplatin, most of which were target agents. There were 3 cases with the type of chemotherapy undescribed in the paper. One patient received both chemotherapy and radiotherapy. The western medication used to subside symptoms were narcotic analgesics (oxycodone, fentanyl patch, and codein), antibiotics, steroids (dexamethasone), diuretics (mannitol), mucolytics (erdosteine) and the medication for urinary incontinence (propiverine), for bronchitis (morniflumate), and for psychosis (quetiapine). Other medications were 
antidiabetics and antihypertension drugs for underlying diseases, and vitamins $\mathrm{B}$ and $\mathrm{C}$ for anticancer purpose.

Analyzing the types of KM treatment, all case reports used herbal medicine except 2 studies. The most frequently used was the allergen-removed Rhus verniciflua Stokes (aRVS, $n=13$ ), followed by HangAmDan (HAD, n=5), HamgAm-Plus (HAP, n=5), Soram-Dan $(n=2)$, Samchilchoongcho-jung $(n=2)$, and other prescriptions to improve certain symptom bojung-ikki-tang, maekmundong-tang, gumsuyukunjun, and so forth. Acupuncture was used in 17 studies, and the mostly used acupuncture points were LI4, LR3, ST36, BL13, and BL17; lung-tonifying acupuncture of Sa-am acupuncture was used in 2 cases; electroacupuncture (EA) was used in 2 cases. Moxibustion was used in 10 studies on su points such as CV12, CV4, CV8, BL13, and BL14. Pharmacopuncture was used in 10 studies which was either intravenous or on acupoints, and the types of herbal medicine included cultivated wild ginseng, Cordyceps militaris, Trichosanthes kirilowii, Carthamus tinctorius, prunella vulgaris, human placenta, and sweet bee venom. Other types of treatment were cupping, meditation, light therapy, aromatherpy, sitz bath, exercise treatment, herbal hot pack, and foot bath herbal massage. Details of each treatment are in the Table 1. (Table 1)

Table 1. The Characteristics of Patients and Interventions in the Included Studies.

\begin{tabular}{|c|c|c|}
\hline Study & Patients & Intervention \\
\hline Park, ${ }^{45)}$ & $\begin{array}{l}1 \text { case of NSCLC patient } \\
\text { (SCC, T4N2M0, StageIIIb) }\end{array}$ & $\begin{array}{l}\text { 1. Herbal medicine (no description) } \\
\text { 2. Pharmacopuncture (clutivated wild ginseng) } \\
\text { 3. Acupuncture (no description for acupoint) }\end{array}$ \\
\hline $\mathrm{Lee}^{48)}$ & $\begin{array}{l}1 \text { case of LC patient with brain } \\
\text { metastasis }\end{array}$ & $\begin{array}{l}\text { 1. Herbal medicine (Seonghyangjeonggi-san with Oryung-san, } \\
\text { Younggyechulgam-tang) } \\
\text { 2. Acupuncture (acupoint for stroke) }\end{array}$ \\
\hline $\mathrm{Cha}^{47)}$ & $\begin{array}{l}1 \text { case of NSCLC patient (SCC, stage } \\
\text { IIIa) }\end{array}$ & $\begin{array}{l}\text { 1. Herbal medicine (Gilgyung-tang) } \\
\text { 2. Light therapy } \\
\text { 3. Pharmacopuncture (Carthamus tinctorius L., Houttuynia cordata Thunberg) } \\
\text { 4. Acupuncture (no description for acupoint) } \\
\text { 5. Moxibustion }\end{array}$ \\
\hline Park, ${ }^{48)}$ & $\begin{array}{c}1 \text { case of LC patient with metastatic } \\
\text { LN (R/O stage III) }\end{array}$ & $\begin{array}{l}\text { 1. Herbal medicine (Gunchilgyebok-Jung/ Gamiyangwe-tang, } \\
\text { Bangpoongtongsung-san) } \\
\text { 2. Acupuncture (LI4, LR3, CV12, ST25) }\end{array}$ \\
\hline Choi, ${ }^{49)}$ & $\begin{array}{c}1 \text { case of NSCLC patient } \\
(\text { adenocarcinoma, Stage IV, EGFR } \\
(+))\end{array}$ & $\begin{array}{l}\text { 1. Herbal medicine (Nobongsangki-Jeong: Loranthus parasticus Merrill, Vespae } \\
\text { Nidus) } \\
\text { 2. Acupuncture (Ashi point) }\end{array}$ \\
\hline Lee $^{50)}$ & $\begin{array}{l}1 \text { case of NSCLC patient with brain } \\
\text { metastasis (T2N1M1) }\end{array}$ & 1. Herbal medicine (Banhabaekchulchenma-tang with Rhus Verniciflua Stokes) \\
\hline Park, $^{51)}$ & $\begin{array}{l}1 \text { case of NSCLC patient me } \\
\text { metastasized to multiple LNs, bone, and } \\
\text { chest wall (adenocarcinoma, stage IV) }\end{array}$ & $\begin{array}{l}\text { 1. Herbal medicine (Yieumjeongajibang/ Gongjin-dan, Bojungyikki-tang, } \\
\text { Yijin-tang, Heangso-san) } \\
\text { 2. Acupuncture (LI4, LR3, ST 36, trigger point at neck) }\end{array}$ \\
\hline $\mathrm{Ha},{ }^{52)}$ & $\begin{array}{l}1 \text { case of NSCLC patient } \\
\text { (adenocarcinoma, stage IV) }\end{array}$ & $\begin{array}{l}\text { 1. Herbal medicine (Samchilchoongcho-Jung: Panax notoginsentg Radix, } \\
\text { Cordyceps militaris, Panax ginseng C.A., Meyer, Boswellia carterii } \\
\text { BIRDWOOD) }\end{array}$ \\
\hline Park, ${ }^{53)}$ & $\begin{array}{l}1 \text { case of NSCLC patient } \\
\quad \text { (SCC, stage IIIa) }\end{array}$ & $\begin{array}{l}\text { 1. Pharmacopuncture (Pulsatilla koreana Nakai, Gingseng Radicella, } \\
\text { Glycyrrhiza uralensis Fischer) }\end{array}$ \\
\hline Park, ${ }^{54)}$ & $\begin{array}{l}1 \text { case of NSCLC patient with bone } \\
\text { and brain metastasis }\end{array}$ & $\begin{array}{l}\text { 1. Herbal medicine } \\
\text { (Samul-tanggagambang with Rhus Verniciflua Stokes) }\end{array}$ \\
\hline
\end{tabular}




\begin{tabular}{|c|c|c|}
\hline Study & Patients & Intervention \\
\hline Bang, ${ }^{55)}$ & $\begin{array}{l}2 \text { case of NSCLC patient } \\
\text { (stage IIIb T2N3 adenocarcinoma; } \\
\text { stage IIIa T1N2) }\end{array}$ & $\begin{array}{l}\text { 1. Pharmacopuncture (Cultivated wild ginseng) } \\
\text { 2. Herbal medicine (Hangam-dan) }\end{array}$ \\
\hline $\mathrm{Kim},{ }^{56)}$ & $\begin{array}{l}1 \text { case of NSCLC patient } \\
\text { (stage IIb T2bN1, SCC) }\end{array}$ & $\begin{array}{l}\text { 1. Pharmacopuncture (Cultivated wild ginseng) } \\
\text { 2. Acupuncture (LI4, LR3, ST36, CV12, ST25) } \\
\text { 3. Cupping } \\
\text { 4. Moxibustion (CV12, BL26) }\end{array}$ \\
\hline Park, ${ }^{57)}$ & $\begin{array}{l}1 \text { case of SCLC patient with brain } \\
\text { metastasis }\end{array}$ & $\begin{array}{l}\text { 1. Herbal medicine (Soeumin Palmulgunja-tang, Jayoon-tang, Soeumin } \\
\text { Jihwangbaekho-tang, Soyangin Dojukganggi-tang, Soyangin } \\
\text { Yangkuksanghwa-tang) }\end{array}$ \\
\hline Choi, ${ }^{58)}$ & 1 case of SCLC patient & $\begin{array}{l}\text { 1. Herbal medicine } \\
\text { 1) Hangam-dan: Coicis Semen, Panax notoginseng Radix, Hippocampus } \\
\text { Kelloggii, Cordyceps Militaris, Santsigu Tuber, Ginseng Radix, Bovis } \\
\text { Calculus, Margarita, and Moschus } \\
\text { 2) PSM (Polysaccharide of mushrooms): Ganodema Lucidum, Hericium } \\
\text { erinaceus, Coriolus versicolor, Grifola frondosa, Lentinus edodes, } \\
\text { Cordyceps militaris, Polyporus umbellatus, Pleurotus ostreatus }\end{array}$ \\
\hline Hong, ${ }^{59)}$ & 1 case of SCLC patient (T3N3M0) & $\begin{array}{l}\text { 1 ElectroAcupuncture (EX-B2 at T5 T7) } \\
\text { 2. Herbal medicine (Samchulgunbi-tang, Bojungyikki-tang) } \\
\text { 3. Acupuncture (Ex-HN3, LU3, LU5, L14, ST36, LR3 } \\
\text { 4. Interferential current therapy (Right flank) }\end{array}$ \\
\hline $\mathrm{Kim}^{60)}$ & $\begin{array}{l}1 \text { case of NSCLC patient } \\
\text { (adenocarcinoma, T4N1M1) }\end{array}$ & $\begin{array}{l}\text { 1. Moxibustion (BL13, BL17, BL42, BL43) } \\
\text { 2. Herbal medicine } \\
\text { 1) Anti-cancer effect: Rhus Verniciflua Stokes } \\
\text { 2) Symptom improvement: Guibi-tang, Shipjeondaebo-tang, yangsayukgunja-tang } \\
\text { 3. Acupuncture (no description for acupoint) }\end{array}$ \\
\hline Song, ${ }^{61)}$ & $\begin{array}{l}1 \text { case of NSCLC patient with bone } \\
\text { metastasis (SCC, stage IV) }\end{array}$ & 1.Herbal medicine (Rhus Verniciflua Stokes) \\
\hline Kwon, ${ }^{62)}$ & $\begin{array}{l}2 \text { cases of NSCLC patients } \\
\text { (stage IV, T4N2M1; stage IIIb, } \\
\text { T4N3M0, adenocarcinoma) }\end{array}$ & $\begin{array}{l}\text { 1.Herbal medicine } \\
\text { 1) Anti-cancer effect: Rhus Verniciflua Stokes } \\
\text { 2) Symptom improvement: Haengsochunggi-tang, Bojungyikki-tang, } \\
\text { Gagamwiryung-tang }\end{array}$ \\
\hline $\mathrm{Yu},{ }^{63)}$ & $\begin{array}{l}1 \text { case of NSCLC patient } \\
\text { (stage IV) }\end{array}$ & $\begin{array}{l}\text { 1. Herbal medicine } \\
\text { 1) Anti-cancer effect: Rhus Verniciflua Stokes } \\
\text { 2) Symptom improvement: unknown Herbal medicne }\end{array}$ \\
\hline Choi, ${ }^{64)}$ & $\begin{array}{l}1 \text { case of NSCLC patient } \\
\quad \text { (adenocarcinoma) }\end{array}$ & $\begin{array}{l}\text { 1. Herbal medicine } \\
\text { 1) Anti-cancer effect: Rhus Verniciflua Stokes } \\
\text { 2) Symptom improvement: Bojungyikki-tang, Boikyangwi-tang, } \\
\text { Banhabaekchulcheonma-tang, Gyuntongdadam-tang }\end{array}$ \\
\hline Park, ${ }^{65)}$ & $\begin{array}{c}1 \text { case of NSCLC patient } \\
\text { (adenocarcinoma, T2N0M1a, stage IV) }\end{array}$ & $\begin{array}{l}\text { 1. Herbal medicine } \\
\text { 1) Rhus Verniciflua Stokes } \\
\text { 2) Bojungyikki-tang, Geumsuyukgunjeon, Sojadodamganggi-tang }\end{array}$ \\
\hline $\mathrm{Kim},{ }^{66)}$ & $\begin{array}{l}1 \text { case of NSCLC patient } \\
\text { (SCC, stage IV) }\end{array}$ & 1. Herbal medicine (Rhus Verniciflua Stokes) \\
\hline Kang, ${ }^{67)}$ & $\begin{array}{l}1 \text { case of SCLC patient \& } 1 \text { case of } \\
\text { HCC patient with lung metastasis }\end{array}$ & $\begin{array}{l}\text { 1. Herbal medicine } \\
\text { 1) Hangam-dan: Coicis Semen, Panax notoginseng Radix, Hippocampus } \\
\text { Kelloggii, Cordyceps Militaris, Santsigu Tuber, Ginseng Radix, Bovis } \\
\text { Calculus, Margarita, and Moschus } \\
\text { 2) PSM (Polysaccharide of mushrooms): Ganodema Lucidum, Hericium } \\
\text { erinaceus, Coriolus versicolor, Grifola frondosa, Lentinus edodes, } \\
\text { Cordyceps militaris, Polyporus umbellatus, Pleurotus ostreatus } \\
\text { 3) Mackmundong-tang, Guibi-tang, Gilgyung-tang } \\
\text { 2. Acupuncture (no description for acupoint) } \\
\text { 3. Moxibustion (no description for acupoint) }\end{array}$ \\
\hline
\end{tabular}




\begin{tabular}{|c|c|c|}
\hline Study & Patients & Intervention \\
\hline Park, ${ }^{68)}$ & $\begin{array}{l}1 \text { case of NSCLC patient with brain } \\
\text { metastasis } \\
\text { (RUL, stage IV) }\end{array}$ & $\begin{array}{l}\text { 1. Herbal medicine } \\
\text { 1) Hangam-dan: Coicis Semen, Panax notoginseng Radix, Hippocampus } \\
\text { Kelloggii, Cordyceps Militaris, Santsigu Tuber, Ginseng Radix, Bovis } \\
\text { Calculus, Margarita, and Moschus } \\
\text { 2) PSM (Polysaccharide of mushrooms): Ganodema Lucidum, Hericium } \\
\text { erinaceus, Coriolus versicolor, Grifola frondosa, Lentinus edodes, } \\
\text { Cordyceps militaris, Polyporus umbellatus, Pleurotus ostreatus } \\
\text { 3) Chunggan extract (CGX): Artemisia capillaris Herba, TrionycisCarapax, } \\
\text { Raphani Semen, Atractylodis Macrocephalae Rhizoma, Poria, Alismatis } \\
\text { Rhizoma, Atractylodis Rhizoma, Salviae Miltiorrhizae Radix,Polyporus, } \\
\text { Amomi Fructus, Aurantii Fructus, Glycyrrhizae Radix or Helenii Radix } \\
\text { 2. Acupuncture (CV-6, CV-15, LR-14, GV-20, LU-11,HT-7, LU-10, GB-24, } \\
\text { LU-1, LU-9, LR-3, LU-6 and BL-63) } \\
\text { 3. Moxibustion (CV-4, HT-8, and KT-1.) }\end{array}$ \\
\hline $\mathrm{Kim}^{27)} 2015$ & $\begin{array}{l}1 \text { case of NSCLC patient with LN } \\
\text { metastasis } \\
\text { (SCC, stage IIIb) }\end{array}$ & $\begin{array}{l}\text { 1. Herbal medicine (Saengmaecksan with baekhapgogeum-tang) } \\
\text { 2. Acupuncture (BL13, BL43, BL23, CV22, LU1, LI4, ST40, LR3, SP3 } \\
\text { 3. Moxibustion (BL26) } \\
\text { 4. Cupping (Su points) } \\
\text { 5. Interferential current therapy (Shoulder and back) } \\
\text { 6. Sitz bath }\end{array}$ \\
\hline $\mathrm{Kim},{ }^{69)}$ & $\begin{array}{c}1 \text { case of NSCLC (adenocarcinoma, } \\
\text { stage IV) }\end{array}$ & $\begin{array}{l}\text { 1. Herbal medicine (Rhus Verniciflua Stokes) } \\
\text { 2. Acupuncture (no description for acupoint) }\end{array}$ \\
\hline Park, ${ }^{70)}$ & 1 case of SCLC patient (limited stage) & $\begin{array}{l}\text { 1. Herbal medicine (Samchilchoongcho-Jung: Panax notoginsentg Radix, } \\
\text { Cordyceps militaris, Panax ginseng C.A., Meyer, Boswellia carterii } \\
\text { BIRDWOOD) }\end{array}$ \\
\hline Zheng, ${ }^{71)}$ & $\begin{array}{c}3 \text { cases of NSCLC patients } \\
\text { (adenocarcinoma, stage IIIb, IV) }\end{array}$ & $\begin{array}{l}\text { 1. Herbal medicine (Atractylodes macrocephala Koidzumi, Houttuyniae Herba, } \\
\text { Zingiber officinale, Farfarae Flos, Stemonae Radix, Areca catechu L } \\
\text { Pueraria lobata Ohwi, Prunus armeniaca var. ansu Maxim, Fritillaria } \\
\text { verticillata var. thunbergii BAK.) }\end{array}$ \\
\hline Choi, $^{72)}$ & 1 case of LC & $\begin{array}{l}\text { 1. Herbal medicine (Hang-Am Plus: Coix lachryma-jobi seed, Panax notoginseng } \\
\text { root, Hippocampus kelloggi, Cordyceps militaris, Cremastra appendiculata } \\
\text { tuber, Panax ginseng root, Bos taurus calculus, Pteria martensii, and Moschus } \\
\text { moschiferus) }\end{array}$ \\
\hline Park, ${ }^{73)}$ & $\begin{array}{l}1 \text { case of NSCLC patient } \\
\text { (adenocarcinoma, stage IIIb, } \\
\text { T2aN0M1a) }\end{array}$ & $\begin{array}{l}\text { 1. Herbal medicine (Hang-Am plus, Samul-tanggagam) } \\
\text { 2. Acupuncture (LI4, LI10, LI11, LR3), Electoracupuncture } \\
\text { 3. Moxibustion (ST25, CV4, CV8, CV12 PC8, KI1) } \\
\text { 4. Herbal hot pack (ST25, CV4, CV8, CV12) } \\
\text { 5. Foot bath Herbal-massage }\end{array}$ \\
\hline $\mathrm{Gu},{ }^{74)}$ & $\begin{array}{l}1 \text { case of LC patient with brain } \\
\text { metastasis (T2N3M1a) }\end{array}$ & $\begin{array}{l}\text { 1. Acupuncture ( LU9, SP3, HT8, LU10) } \\
\text { 2. Herbal medicine (Cheongpebyeolgap-san, Ssangpae-tang, Yangwijinsik-tang, } \\
\text { Shigyunghwadamjeon) } \\
\text { 3. Meditation } \\
\text { 4. Moxibustion (CV12, CV4) } \\
\text { 5. Cupping (Su points) } \\
\text { 6. Pharmacopuncture (Carthamus tinctorius L, Hominis Placenta, Sweet bee } \\
\text { venom) }\end{array}$ \\
\hline Choi, ${ }^{75)}$ & 1 case of SCLC patient (T3M2M0) & $\begin{array}{l}\text { 1. Herbal medicine (Hangam-dan: Coicis Semen, Panax notoginseng Radix, } \\
\text { Hippocampus Kelloggii, Cordyceps Militaris, Santsigu Tuber, Ginseng } \\
\text { Radix, Bovis Calculus, Margarita, and Moschus) }\end{array}$ \\
\hline Yoo $^{76)}$ & 1 case of NSCLC patient (SCC) & $\begin{array}{l}\text { 1. Herbal medicine } \\
\text { 1) Hangam-dan: Coicis Semen, Panax notoginseng Radix, Hippocampus } \\
\text { Kelloggii, Cordyceps Militaris, Santsigu Tuber, Ginseng Radix, Bovis } \\
\text { Calculus, Margarita, and Moschus } \\
\text { 2) Pyungpe-eum, Maekmundong-tang }\end{array}$ \\
\hline
\end{tabular}




\begin{tabular}{|c|c|c|}
\hline Study & Patients & Intervention \\
\hline Park, ${ }^{77)}$ & $\begin{array}{l}1 \text { case of NSCLC patient } \\
\text { (adenocarcinoma, stage IV, } \\
\text { cT3N1M1a) }\end{array}$ & 1. Herbal medicine (Taeeumin Galgeunhaegi-tanggami) \\
\hline $\mathrm{Kim}^{78)}$ & $\begin{array}{c}1 \text { case of NSCLC patient } \\
\text { (adenocarcinoma, stage IV, T4NOMx) } \\
\& 3 \text { cases of NSCLC patients (SCC, } \\
\text { stage IIIb, IV) }\end{array}$ & 1. Herbal medicine (Hang-am plus) \\
\hline Lee, ${ }^{79)}$ & $\begin{array}{l}1 \text { case of NSCLC patient } \\
\text { (adenocarcinoma, stage IV, } \\
\text { cT1N2M1) }\end{array}$ & 1. Herbal medicine (Rhus Verniciflua Stokes) \\
\hline Son, ${ }^{80)}$ & $\begin{array}{c}1 \text { case of } \mathrm{LC} \\
\text { (stage } \mathrm{IIIb}, \mathrm{T} 3 \mathrm{~N} 1 \mathrm{M} 0 \text { ) }\end{array}$ & $\begin{array}{l}\text { 1. Acupuncture (LU9, SP3, HT8, LU10, LU8, KI7, SP3, KI3) } \\
\text { 2. Herbal medicine (Samjahwadamjeon, Mundongcheongpeeum, } \\
\text { Samchulbope-tang) } \\
\text { 3. Moxibustion (BL13, Ashi point) } \\
\text { 4. cupping (Su points, SI11, SI9, CV17) }\end{array}$ \\
\hline $\mathrm{Yi}^{8}{ }^{81)}$ & 1 case of LC patient & 1. Herbal medicine (Geumsoyukgunjeon) \\
\hline Park, ${ }^{82)}$ & $\begin{array}{l}1 \text { case of NSCLC patient } \\
\text { (adenocarcinoma, } \\
\text { stage IV, T1N0M1) }\end{array}$ & $\begin{array}{l}\text { 1. Herbal medicine } \\
\text { 1) Hang Am Plus: Coix lachryma-jobi seed, Panax notoginseng root, } \\
\text { Hippocampus kelloggi, Cordyceps militaris, Cremastra appendiculata tuber, } \\
\text { Panax ginseng root, Bos taurus calculus, Pteria martensii, and Moschus } \\
\text { moschiferus } \\
\text { 2) Myunyeok Plus: Astragalus membranaceus, Salvia miltiorrhiza, Hericium } \\
\text { erinaceum, and Panax ginseng } \\
\text { 3) Soshiho-tang, Soyo-san, Dohongsamul-tang, Banhasashim-tang } \\
\text { 2. Acupuncture (SP02, HT08, SP01, LR01, SP03, LU09, TE06, KI02, LU08, } \\
\text { SP03, HT08, CV06, BL15) } \\
\text { 3. Pharmacopuncture (Bee venom and placenta pharmacopuncture were used in } \\
\text { turns on the acupoints CV12 and GV3) } \\
\text { 4. Physical therapy (cupping, TENS, light therapy) } \\
\text { 5. Thermotherapy, hydrotherapy (Moxibustion (CV12 and KD1), foot bath, hot } \\
\text { pack) } \\
\text { 6. Aromatherapy } \\
\text { 7. Massage therapy } \\
\text { 8. Meditation therapy } \\
\text { 9. Supportive therapy } \\
\text { 10. Exercise therapy }\end{array}$ \\
\hline Lee, ${ }^{83)}$ & $\begin{array}{c}13 \text { cases of NSCLC patients (stage } \\
\text { IIIB, IV) }\end{array}$ & 1. herbal medicine (Rhus Verniciflua Stokes) \\
\hline Hong, ${ }^{84)}$ & $\begin{array}{c}1 \text { case of NSCLC patient } \\
\text { (adenocarcinoma, pT1bN0, stage IA2) }\end{array}$ & $\begin{array}{l}\text { 1. herbal medicine (Banhahubak-tang, Gamiondamtang) } \\
\text { 2. acupuncture (EX-HN3, CV17, LU5, LI4, ST36, LR3, SP6, SP3, ST40, GB39, } \\
\text { EX-B2, CV21, CV20, L14) }\end{array}$ \\
\hline Song, ${ }^{85)}$ & 1 case of NSCLC patient (stage IIIC) & $\begin{array}{l}\text { 1. herbal medicine (gami shigyungbanha-tang, sojadodam-tang, } \\
\text { gunggwihyangso-san gamibang, hwaggigyegi-tang gamibang) }\end{array}$ \\
\hline Lee, ${ }^{86)}$ & $\begin{array}{c}1 \text { case of NSCLC patient } \\
\text { (adenocarcinoma, T4N0M0, stage } \\
\text { IIIB) }\end{array}$ & 1. herbal medicine (Rhus Verniciflua Stokes) \\
\hline
\end{tabular}




\begin{tabular}{|c|c|c|}
\hline Study & Patients & Intervention \\
\hline $\mathrm{Kim},{ }^{87)}$ & $\begin{array}{l}1 \text { case of HCC patient with lung } \\
\text { metastases }\end{array}$ & 1. herbal medicine (Rhus Verniciflua Stokes, dokhwaljihwang-tang) \\
\hline Jang, ${ }^{88)}$ & $\begin{array}{l}1 \text { case of HCC patient with lung } \\
\text { metastasis }\end{array}$ & $\begin{array}{l}\text { 1. pharmacopuncture (wild ginseng, cordyceps militaris, prunella vulgaris) } \\
\text { 2. nasal fumigation } \\
\text { 3. herbal medicine (bulhwangeum junggi-san) } \\
\text { 4. moxibustion (CV4, CV8, ST25) } \\
\text { 5. foot bath (herbal medicated, bojungikki-tang }\end{array}$ \\
\hline Lim, ${ }^{89)}$ & $\begin{array}{l}1 \text { case of HCC patient with lung } \\
\text { metastases }\end{array}$ & 1.herbal medicine (Dendropanax morbifera) \\
\hline Lee, ${ }^{90)}$ & $\begin{array}{l}1 \text { case of bladder cancer patient with } \\
\text { lung metastasis }\end{array}$ & $\begin{array}{l}\text { 1. pharmacopuncture (wild ginseng, Cordyceps sinensis, Trichosanthes } \\
\text { kirilowii, Platycodon grandiflorus) } \\
\text { 2. herbal medicine (Soramdan, CS distillate, Gunchildan, Hyunamdan, } \\
\text { Hangamdan S) } \\
\text { 3. Soram nebulizer solution }\end{array}$ \\
\hline Lee, ${ }^{91)}$ & $\begin{array}{l}1 \text { case of NSCLC patient (SCC, stage } \\
\text { IV) }\end{array}$ & $\begin{array}{l}\text { 1. pharmacopuncture (wild ginseng, Cordyceps sinensis, Trichosanthes } \\
\text { kirilowii) } \\
\text { 2. herbal medicine (Soramdan) }\end{array}$ \\
\hline
\end{tabular}

NSCLC: Non-Small Cell Lung Carcinoma, SCC: Squamous Cell Carcinoma, LC: lung Cancer, LN: Lymph Node, EGFR: Epidermal Growth Factor Receptor, SCLC: Small Cell Lung Carcinoma, HCC: Hepatocellular Carcinoma, RUL: Right Upper Lobe

\section{5) Evaluation variables}

(1) Efficacy evaluation variables

Several efficacy evaluation variables were used in each case, and they were tumor size based on Response Evaluation Criteria in Solid Tumors (RECIST), changes of symptoms, duration of survival, the quality of life, tumor markers and so on. The changes of symptoms were not only about the symptoms from cancer itself, but about the side effects from conventional treatment, which was covered in five reports. The instruments for evaluating the quality of life were Eastern Cooperative Oncology Group Performance Status (ECOG PS), Functional Assessment of Cancer Therapy-Lung (FACT-L), Functional Assessment of Chronic Illness Therapy- Fatigue (FACIT-F), European organization for research and treatment of cancer quality of life questionnaire, Core 30 (EORTC-QLQ C30), and EORTC QLQ- lung cancer 13 (EORTC QLQ-LC13). Two cases evaluated metastasized tumor, both of which were about brain metastasis. All the details are provided in the Table 2. (Table 2)
(2) Safety evaluation variables

The safety was evaluated by checking adverse effects using blood test (liver and kidney function test mainly) or N CI-CTCAE. Details are in the table 3.

\section{6) Major results}

As a result of evaluating the tumor size, partial response (PR) was in 12 cases - including "decrease" of tumor size, and stable disease (SD) was in 22 cases - including "no interval change." On the other hand, time to progress (TTP), evaluated by the tumor size based on RECIST, was used in one study. The median TTP was 182 (range, 36-738) days in the study. The duration of survival was measured in 11 case reports, showing extended length of survival than the expectancy of corresponding stage. The quality of life was evaluated in 17 cases, all of which reported improved QOL or stable satisfactory QOL except one case of worsening. Meanwhile, the improvement of symptoms was evaluated in all cases except for two cases. Especially, 6 cases reported the improvement of side effects from chemotherapy or surgery. (Table 2) 
Table 2. The Outcome Measurements and Results of the Included Studies.

\begin{tabular}{|c|c|c|}
\hline Study & Outcome measurements & Results \\
\hline \multicolumn{3}{|l|}{ Tumor size } \\
\hline Park, ${ }^{45)} 2007$ & Tumor size (Chest-CT) & $\begin{array}{l}\text { 1. Size of tumor stabilized. } \\
\text { 2. Pleural effusion decreased. }\end{array}$ \\
\hline Park, ${ }^{48)} 2015$ & $\begin{array}{l}\text { 1. Symptoms (NRS) } \\
\text { 2. LFT } \\
\text { 3. Chest CT }\end{array}$ & $\begin{array}{l}\text { 1. NRS decreased. } \\
\text { 2. LFT stabilized. } \\
\text { 3. Tumor size slightly decreased. }\end{array}$ \\
\hline $\mathrm{Ha}^{52)} 2018$ & $\begin{array}{l}\text { 1. Tumor size (RECIST) } \\
\text { 2. NCI-CTCAE } \\
\text { 3. Blood test } \\
\text { 4. ECOG PS } \\
\text { 5. Symptom }\end{array}$ & $\begin{array}{l}\text { 1. Stable disease } \\
\text { 2. No change in NCI-CTCAE grade } \\
\text { 3. No change in LFT; tumor marker stabilized or slightly decreased. } \\
\text { 4. ECOG PS grade lowered. }\end{array}$ \\
\hline Park, ${ }^{53)} 2014$ & $\begin{array}{l}\text { 1. Symptom } \\
\text { 2. Blood test } \\
\text { 3. Tumor size (Chest CT) }\end{array}$ & $\begin{array}{l}\text { 1. Cough and pain improved. } \\
\text { 2. Total protein, albumin, r-GTP, CRP, WBC and PLT stabilized. } \\
\text { 3. Tumor size decreased; pneumonia diminished. }\end{array}$ \\
\hline Bang, ${ }^{55)} 2008$ & $\begin{array}{l}\text { 1. Symptoms } \\
\text { 2. Tumor size (Chest CT, PET-CT) }\end{array}$ & $\begin{array}{l}\text { 1. Symptoms improved. } \\
\text { 2. Stable disease }\end{array}$ \\
\hline $\mathrm{Kim},{ }^{56)} 2011$ & $\begin{array}{l}\text { 1. Tumor size (Chest CT) } \\
\text { 2. Symptom }\end{array}$ & $\begin{array}{l}\text { 1. Tumor size decreased. } \\
\text { 2. Dyspnea improved }\end{array}$ \\
\hline $\mathrm{Kim}^{27)} 2015$ & $\begin{array}{l}\text { 1. Tumor size (Chest CT) } \\
\text { 2. ECOG PS }\end{array}$ & $\begin{array}{l}\text { 1. Decreased mass size } \\
\text { 2. Stable ECOG PS }\end{array}$ \\
\hline $\mathrm{Kim},{ }^{69)} 2017$ & $\begin{array}{l}\text { 1. NRS } \\
\text { 2. Tumor size RECIST } \\
\text { 3. NCI-CTCAE } \\
\text { 4. Blood test } \\
\text { 5. QoL (ECOG PS) }\end{array}$ & $\begin{array}{l}\text { 1. Improved NRS } \\
\text { 2. Stable disease } \\
\text { 3. Stable NCI-CTCAE grade } \\
\text { 4. Stable LFT; decreased tumor marker } \\
\text { 5. Improved ECOG PS }\end{array}$ \\
\hline Park, ${ }^{70)} 2005$ & $\begin{array}{l}\text { 1. Tumor size (Chest XR, CT) } \\
\text { 2. Symptoms }\end{array}$ & $\begin{array}{l}\text { 1. Partial response } \\
\text { 2. Symptoms improved }\end{array}$ \\
\hline Zheng, ${ }^{71)} 2011$ & $\begin{array}{l}\text { 1. Tumor size (Chest CT) } \\
\text { 2. VAS } \\
\text { 3. ECOG PS (QoL) } \\
\text { 4. Blood test }\end{array}$ & $\begin{array}{l}\text { [CASE 1] } \\
\text { 1. Stable disease } \\
\text { 2. No change in VAS score } \\
\text { 3. Stable ECOG PS } \\
\text { 4. Stable LFT and kidney function } \\
\text { [CASE 2] } \\
\text { 1. Stable disease; aggravation of pleural effusion } \\
\text { 2. Decrease in VAS score } \\
\text { 3. Stable ECOG PS } \\
\text { 4. Stable LFT and kidney function } \\
\text { [CASE 3] } \\
\text { 1. Stable disease } \\
\text { 2. Decrease in VAS score } \\
\text { 3. Aggravation of ECOG PS } \\
\text { 4. Stable LFT and kidney function }\end{array}$ \\
\hline Choi, ${ }^{75)} 2005$ & $\begin{array}{l}\text { 1. Tumor size (Chest XR) } \\
\text { 2. survival }\end{array}$ & $\begin{array}{l}\text { 1. Partial response } \\
\text { 2. } 8 \text { years of survival }\end{array}$ \\
\hline $\mathrm{Kim},{ }^{78)} 2009$ & $\begin{array}{l}\text { 1. Tumor size (Chest CT) } \\
\text { 2. symptoms }\end{array}$ & $\begin{array}{l}\text { [CASE 1] } \\
\text { 1. Stable disease } \\
\text { 2. Improved symptoms } \\
\text { [CASE 2] } \\
\text { 1. Increase of tumor size (decrease } \rightarrow \text { increase) }\end{array}$ \\
\hline
\end{tabular}




\begin{tabular}{|c|c|c|}
\hline Study & Outcome measurements & Results \\
\hline $\mathrm{Kim}^{78)} 2009$ & $\begin{array}{l}\text { 1. Tumor size (Chest CT) } \\
\text { 2. symptoms }\end{array}$ & $\begin{array}{l}\text { 2. Improved symptoms } \\
\text { [CASE 3] } \\
\text { 1. Stable disease } \\
\text { 2. Improved symptoms } \\
\text { [CASE 4] } \\
\text { 1. Increase } \rightarrow \text { stable disease } \\
\text { 2. Improved symptoms }\end{array}$ \\
\hline Lee $^{83)} 2009$ & $\begin{array}{l}\text { 1. Time to progression (TTP) } \\
\text { 2. CTCAE }\end{array}$ & $\begin{array}{l}\text { 1. TTP median } 183 \\
\text { 2. no significant } \mathrm{AE}\end{array}$ \\
\hline Lee, ${ }^{86)} 2009$ & $\begin{array}{l}\text { 1. Tumor size (Chest CT) } \\
\text { 2. ECOG PS }\end{array}$ & $\begin{array}{l}\text { 1. Slight decrease in tumor size and loss of pleural effusion. } \\
\text { 2. ECOG PS maintained. }\end{array}$ \\
\hline $\mathrm{Kim},{ }^{87)} 2016$ & $\begin{array}{l}\text { 1. Tumor size (Chest CT) } \\
\text { 2. ECOG PS } \\
\text { 3. progression-free survival (PFS) }\end{array}$ & $\begin{array}{l}\text { 1. Lung metastases disappeared } \\
\text { 2. ECOG PS improved } \\
\text { 3. Over } 25 \text { months of PFS (출판시까지) }\end{array}$ \\
\hline Jang, ${ }^{88)} 2018$ & $\begin{array}{l}\text { 1. Tumor size (Chest CT) } \\
\text { 2. Tumor marker (CA19-9) } \\
\text { 3. Blood test }\end{array}$ & $\begin{array}{l}\text { 1. Reduction in size of metastatic lodules in lungs } \\
\text { 2. Tumor marker decreased } \\
\text { 3. Decreased AST and LDH, normal ALT, ALP, t-bil, BUN, Cr }\end{array}$ \\
\hline $\mathrm{Lim}^{89)} 2014$ & $\begin{array}{l}\text { 1. Tumor size (Chest XR, CT) } \\
\text { 2. Tumor marker (AFP, PIVKA II) } \\
\text { 3. Symptoms }\end{array}$ & $\begin{array}{l}\text { 1. Lung metastases disappeared } \\
\text { 2. AFP and PIVKA II level decreased. } \\
\text { 3. Symptoms improved }\end{array}$ \\
\hline Lee, $^{90)} 2014$ & 1. Tumor size (Chest XR) & 1.tumor size increase $->$ nearly disappeared \\
\hline \multicolumn{3}{|l|}{ Symptom } \\
\hline Lee, ${ }^{46)} 1999$ & $\begin{array}{l}\text { 1. Brain CT/MRI } \\
\text { 2. Chest XR or CT } \\
\text { 3. Muscle test (Lakin) }\end{array}$ & $\begin{array}{l}\text { 1. Motor and urination was improved. } \\
\text { 2. No change in brain-CT. }\end{array}$ \\
\hline Cha, ${ }^{47)} 1997$ & $\begin{array}{l}\text { 1. Blood test (ALP) } \\
\text { 2. Symptoms } \\
\text { 3. Chest XR, MRI }\end{array}$ & $\begin{array}{l}\text { 1. ALP level decreased. } \\
\text { 2. Hemoptysis stopped. } \\
\text { 3. Tumor size stabilized } \\
\text { 4. Improved pneumonia }\end{array}$ \\
\hline Lee, ${ }^{50)} 2009$ & $\begin{array}{l}\text { 1. NCI-CTCAE (headache) } \\
\text { 2. VAS (headache) }\end{array}$ & $\begin{array}{l}\text { 1. NCI-CTCAE grade lowered. } \\
\text { 2. VAS decreased. }\end{array}$ \\
\hline Park, ${ }^{51)} 2014$ & $\begin{array}{l}\text { 1. Symptoms (VAS) } \\
\text { 2. Blood test } \\
\text { 3. Chest XR }\end{array}$ & $\begin{array}{l}\text { 1. VAS score decreased. } \\
\text { 2. LFT stabilized; WBC, neutrophil, and CEA decreased. } \\
\text { 3. Pleural effusion improved. }\end{array}$ \\
\hline Park, ${ }^{57)} 2014$ & 1. Symptoms & 1. Delirium, constipation, and appetite improved. \\
\hline Choi ${ }^{58)} 2003$ & $\begin{array}{l}\text { 1. Symptoms } \\
\text { 2. Chest PA, CT } \\
\text { 3. Blood test }\end{array}$ & $\begin{array}{l}\text { 1. Symptoms improved. } \\
\text { 2. NIC } \rightarrow \text { meta to RLL } \rightarrow \text { improvement of RLL } \rightarrow \text { NIC } \\
\text { 3. Total protein and } \mathrm{Hb} \text { stabilized. }\end{array}$ \\
\hline Hong, ${ }^{59)} 2014$ & 1. NRS & 1. NRS score lowered. \\
\hline $\mathrm{Kim},{ }^{60)} 2009$ & $\begin{array}{l}\text { 1. Symptoms (BPI, VAS) } \\
\text { 2. Analgesic dose }\end{array}$ & $\begin{array}{l}\text { 1. Symptoms improved. } \\
\text { 2. Analgesic dose reduction }\end{array}$ \\
\hline Kwon, ${ }^{62)} 2009$ & $\begin{array}{l}\text { 1. Chest CT } \\
\text { 2. ECOG PS(survival extended) }\end{array}$ & $\begin{array}{l}{[\mathrm{CASE} \text { 1] }} \\
\text { 1. Progression } \rightarrow \text { NIC } \\
\text { 2. ECOG PS improved } \\
{[\mathrm{CASE} \text { 2] }} \\
\text { 1. PR } \rightarrow \text { no remarkable change } \\
\text { 2. Stable ECOG PS }\end{array}$ \\
\hline
\end{tabular}




\begin{tabular}{|c|c|c|}
\hline Study & Outcome measurements & Results \\
\hline $\mathrm{Gu}^{74)} 2011$ & $\begin{array}{l}\text { 1. BDI, HRSD } \\
\text { 2. BAI, ASI } \\
\text { 3. Symptoms }\end{array}$ & $\begin{array}{l}\text { 1. Improved depression } \\
\text { 2. Improved anxiety } \\
\text { 3. Improved symptoms }\end{array}$ \\
\hline Son, ${ }^{80)} 2009$ & $\begin{array}{l}\text { 1. Chest XR } \\
\text { 2. Symptoms } \\
\text { 3. QLQAKA }\end{array}$ & $\begin{array}{l}\text { 1. No change in tumor size; haziness and lobulated pleural lesion } \\
\text { improved. } \\
\text { 2. Improved symptoms } \\
\text { 3. Improved QLQAKA }\end{array}$ \\
\hline $\mathrm{Yi}^{81)} 2003$ & 1. Symptoms & 1. Improved symptoms \\
\hline Song, ${ }^{85)} 2017$ & $\begin{array}{l}\text { 1. Chest XR } \\
\text { 2. inflammation markers (WBC, neutrophil } \\
\text { counts, ESR, CRP, procalcitonin) } \\
\text { 3. symptoms }\end{array}$ & $\begin{array}{l}\text { 1. Chest XR improved } \\
\text { 2. inflammation marker level decreased. } \\
\text { 3. symptoms improved. }\end{array}$ \\
\hline \multicolumn{3}{|l|}{ QoL } \\
\hline Song, ${ }^{61)} 2012$ & $\begin{array}{l}\text { 1. QoL } \\
\text { 2. Chest CT, XR } \\
\text { 3. Survival duration }\end{array}$ & $\begin{array}{l}\text { 1. QoL improved } \\
\text { 2. Chest CT result was WNL. } \\
\text { 3. } 15 \text { years of survival (until the time of publication) }\end{array}$ \\
\hline $\mathrm{Yu},{ }^{63)} 2008$ & $\begin{array}{l}\text { 1. Chest CT } \\
\text { 2. QoL } \\
\text { 3. Blood test }\end{array}$ & $\begin{array}{l}\text { 1. Slight increase in tumor size } \\
\text { 2. QoL improved } \\
\text { 3. Stable LFT }\end{array}$ \\
\hline Park, ${ }^{68)} 2011$ & $\begin{array}{l}\text { 1. VAS, FACT-L, FACIT-F } \\
\text { 2. Chest CT }\end{array}$ & $\begin{array}{l}\text { 1. Increased VAS; stable FACT-L, FACIT-F (improvement for } 1 \\
\text { week) } \\
\text { 2. Decrease of pleural effusion; aggravation of pneumonia }\end{array}$ \\
\hline Park, ${ }^{82)} 2010$ & $\begin{array}{l}\text { 1. Survival } \\
\text { 2. Chest CT } \\
\text { 3. Symptoms }\end{array}$ & $\begin{array}{l}\text { 1. Disease-free survival for } 28 \text { months without adjuvant chemotherapy. } \\
\text { 2. Neither metastasis nor recurrence occurred during this period. } \\
\text { 3. Exertional dyspnea recovered to NYHA grade 1. Other physical and } \\
\text { psychological symptoms were alleviated. }\end{array}$ \\
\hline Lee, ${ }^{91)} 2013$ & $\begin{array}{l}\text { 1. ECOG PS } \\
\text { 2. Tumor size (Chest CT) }\end{array}$ & $\begin{array}{l}\text { 1.ECOS PS improved } \\
\text { 2.increased size } \rightarrow \text { decreased size } \rightarrow \text { stable disease }\end{array}$ \\
\hline
\end{tabular}

Adverse event due to conventional treatment

\begin{tabular}{|c|c|c|}
\hline Choi ${ }^{49)} 2017$ & $\begin{array}{l}\text { 1. NRS (skin rash) } \\
\text { 2. NCI-CTCAE } \\
\text { 3. Blood test (CRP, fibrinogen) }\end{array}$ & $\begin{array}{l}\text { 1. NRS decreased. } \\
\text { 2. NCI-CTCAE grade lowered. } \\
\text { 3. CRP and fibrinogen stabilized. }\end{array}$ \\
\hline Park, $^{54)} 2011$ & 1. NCI-CTCAE & 1. NCI-CTCAE grades lowered. \\
\hline Choi, ${ }^{72)} 2010$ & $\begin{array}{l}\text { 1. ROM } \\
\text { 2. Physical examination (HSBT, MWT) } \\
\text { 3. VAS } \\
\text { 4. Abdominal discomfort }\end{array}$ & $\begin{array}{l}\text { 1. Improved ROM } \\
\text { 2. Improved HSBT, stable MWT } \\
\text { 3. Improved VAS score } \\
\text { 4. Improved abdominal discomfort }\end{array}$ \\
\hline Park, $^{73)} 2012$ & $\begin{array}{l}\text { 1. Blood test } \\
\text { 2. VAS } \\
\text { 3. NCI-CTCAE } \\
\text { 3. ECOG PS }\end{array}$ & $\begin{array}{l}\text { 1. Stable LFT and CBC } \\
\text { 2. Improved VAS } \\
\text { 3. Improved NCI-CTCAE grade } \\
\text { 4. Improved ECOG PS }\end{array}$ \\
\hline Park, ${ }^{77)} 2015$ & 1. symptoms(NRS) & 1. Improved symptoms \\
\hline Hong, ${ }^{84)} 2016$ & $\begin{array}{l}\text { 1. EORTC QLQ-C30, LC13 } \\
\text { 2. NRS (pain) } \\
\text { 3. Global assessment (dyspnea) }\end{array}$ & $\begin{array}{l}\text { 1.EORTC QLQ improved } \\
\text { 2.NRS score improved } \\
\text { 3.Global assessment dyspnea improved }\end{array}$ \\
\hline \multicolumn{3}{|c|}{ Survival duration } \\
\hline Choi, ${ }^{64)} 2012$ & $\begin{array}{l}\text { 1. Chest CT } \\
\text { 2. Survival duration }\end{array}$ & 1. No change of tumor size; pleural effusion improved \\
\hline
\end{tabular}




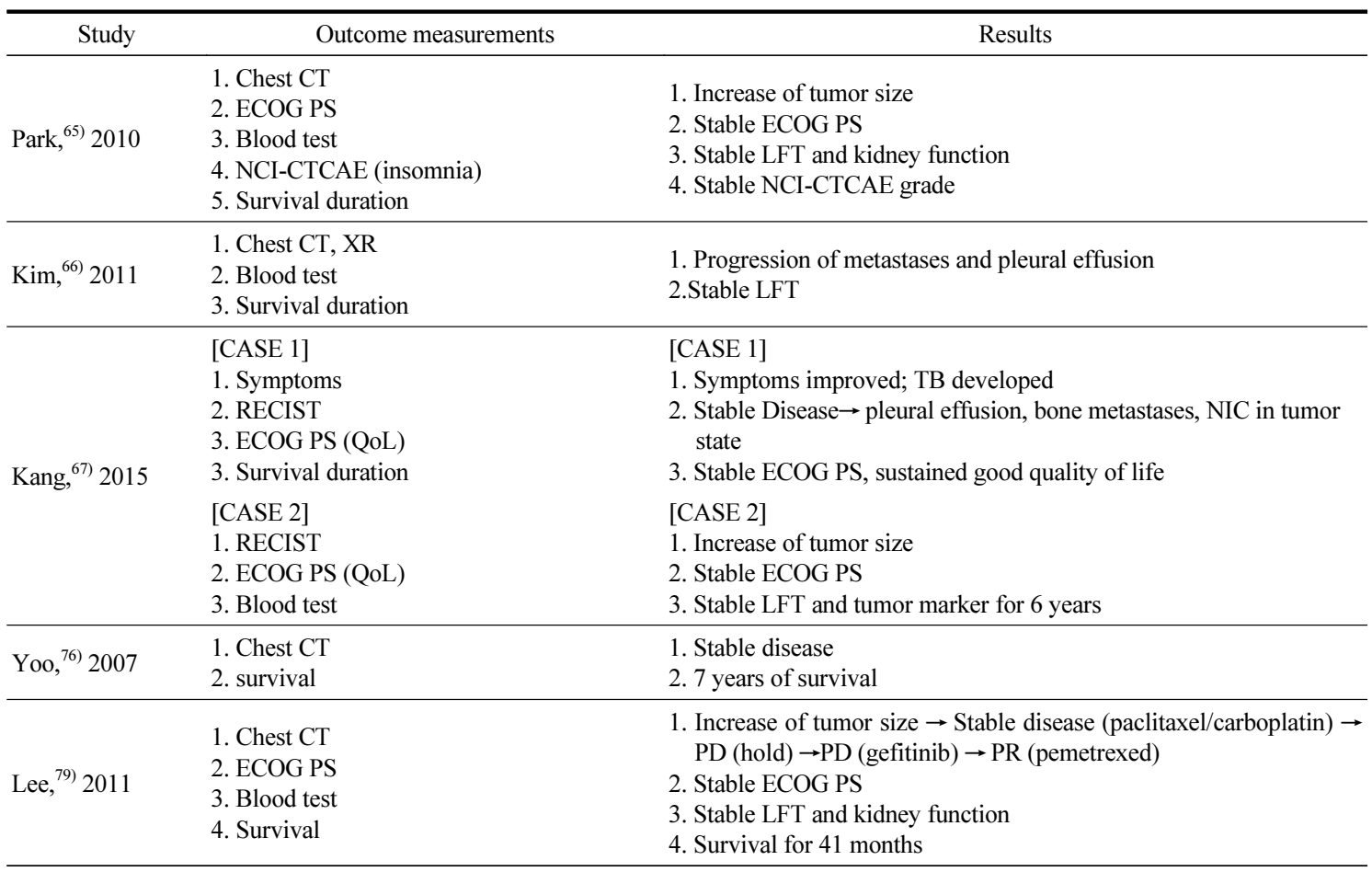

CT: Computed Tomography, MRI: Magnetic Resonance Imaging, XR: X-ray, ALP: Alkaline Phosphatase, NRS: Numeric Rating Scale, LFT: Liver Function Test, NCI-CTCAE: National Cancer Institute - Common Terminology Criteria for Adverse Events, CRP: C-Reactive Protein, VAS: Visual Analogue Scale, WBC: White Blood Cell, CEA: Carcinoembryonic Antigen, RECIST: Response Evaluation Criteria In Solid Tumors, ECOG PS: Eastern Cooperative Oncology Group Performance Status, r-GTP: Gamma-glutamyl transferase, PLT: Platelet, NIC: No Interval Change, RLL: Right Lower lobe, Hb: Hemoglobin, BPI: Brief Pain Inventory, QoL: Quality of Life, WNL: Within Normal Limits, TB: Tuberculosis, FACT-L: Functional Assessment of Cancer Therapy - Lung, FACIT-F: Functional Assessment of Chronic Illness Therapy - Fatigue, ROM: Range of Motion, HSBT: Hand to Shoulder Blade Test, MWT: Mouth Wrap Test, CBC: Complete Blood Cell Count, BDI: Beck's Depression Inventory, HRSD: Hamilton Rating Scale for Depression, BAI: Beck's Anxiety Inventory, ASI: Anxiety Status Inventory, QLQAKA: Quality of Life Questionnaire for Adult Korean Asthmatics

\section{Discussion}

Cancer patients and their family are physically, psychologically, and socio-economically burdened by the side effects and the limitation of conventional treatment. ${ }^{15)}$ For this reason, patients are seeking for complementary and alternative medicine including $\mathrm{KM}$ with the growing demand on cancer treatment of KM, and different types of treatment by KM practitioners are in clinical use indeed. ${ }^{16)}$ However, in the circumstance where no little medical doctors of western medicine prohibit patients from receiving $\mathrm{KM}$ treatment, the barrier to treat cancer patients is quite high for KM doctors currently, so treating cancer in $\mathrm{KM}$ is less generalized than treating other diseases such as musculoskeletal disorders. In consequence, KM doctors frequently search for case reports of similar cases to figure out treatment methods and their effects, obtain knowledge on therapeutic approach of $\mathrm{KM}$ on cancer, and apply them in clinics. The doctors require valid information on diagnosis, prognosis, and prevention in daily clinical practice, but the time to deal with it is comparatively limited. ${ }^{17)}$ Thus we decided an integrative study is needed - which integrates and 
analyzes those individual case reports. Therefore, this study analyzed case reports on lung cancer treated by $\mathrm{KM}$, and reorganized the types of patients visiting $\mathrm{KM}$ clinics, the treatment methods, the result and the prognosis of patients to make it applicable to clinical practice.

The number of studies searched in the international database (PubMed and EMBASE) was fewer than that of articles in the national databases. The reason why case reports on KM are hard to find in the international databases may be related to the phenomenon where the most journals are reluctant to publish case reports because the low citation index of the case report negatively influences the impact factor of the journal. ${ }^{18)}$

Regarding the trend of publishing case reports by year, it has been constant since 1997, and especially in 2011,7 case reports were published at the peak. Only 18 cases were reported in the recent 5 years, taking up less than $38 \%$ of the total, which is rather insufficient compared to the increasing demand on $\mathrm{KM}$ treatment. The domestic journals with the most publication are the journal of Korean traditional oncology and the journal of internal Korean medicine.

The total number of patients reported in the studies were 68 , and except for 5 patient with lung metastasis originated from liver, rectal, and bladder cancer, 63 patients had primary lung cancer. The types of lung cancer included NSCLC in 41 patients and SCLC in 6 patients, with higher percentage of NSCLC patients, because NSCLC takes up $85 \%$ of the lung cancer. ${ }^{19)}$ Among the NSCLC patients were 12 cases of squamous cell carcinoma and 22 cases of adenocarcinoma which is dominant; this result corresponds to the steady trend of increasing percentage of squamous cell carcinoma in Korea as long as the north America and Japan. ${ }^{20)}$ Meanwhile, SCLC were only in 6 cases, half of which did not describe whether limited or extensive stage. In consideration of it, SCLC has not been widely dealt with in clinical $\mathrm{KM}$, and further case reports are to be expected to accumulate.

Advanced cancer with metastasis were found in 40 cases, which accounts for $59 \%$ of the total. This reflects the characteristic of lung cancer; more than $40 \%$ of NSCLC are diagnosed after developing metastasis, which is stage IV, ${ }^{19)}$ and two thirds of SCLC are found with distant metastasis other than chest wall at the point of diagnosis. ${ }^{21)}$ Assuming that the case reports are reflection of virtual clinic, the number of advanced lung cancer patients are considerable, and KM treatment reflecting this clinical reality needs to be established. In clinics, the characteristic of lung cancer to be easily metastasized should be considered when treating patients.

The number of patients under combined standard treatment and KM was 25 in the selected cases reports. The number of patients under KM therapy alone was 40 , for prevention of recurrence/metastasis or treating side effects after finishing standard treatment, due to refusal to receive standard western treatment, or due to old age. More precisely, 6 patients were under periodic follow-up or prevention of recurrence/metastasis or treating side effects after the conventional treatment of surgery, chemotherapy, and radiotherapy; for 8 patients, it was difficult to continue the conventional therapy due to side effects; 1 patient could not receive the western medical treatment due to old age; 3 patients for palliative treatment; and 22 patients refused the conventional treatment. The reasons of refusal were weakened physical strength, old age, concerns about side effects, preference for natural therapy, and experience of aggravation or severe side effects even after conventional treatment. A considerable number of patients refused the conventional treatment, which is frequently seen in the KM hospital specialized for cancer. If the patient is eligible for the standard treatment and is expected to benefit from it, KM therapy alone 
may involve an ethical issue, so the physician should be decided depending on the condition of the patient. Meanwhile, 6 patients were under KM therapy alone to prevent recurrence/metastasis or treat side effects after finishing the conventional treatment. In recent KM clinics, patients with this kind of purpose are emerging, and they complain of anxiety about recurrence/metastasis of residual tumor without any medical treatment after the end of conventional treatment. Not only case reports but also experimental studies on herbal medicine effective in preventing recurrence or metastasis have been published, ${ }^{22)}$, 23) so these are considered to be helpful for patients applied in clinic as evidence.

Targeted therapy was mostly used as chemotherapy combined with KM therapy. This owes to lively carried out genetic research on the mechanism of carcinogenesis of lung cancer, so NSCLC was able to be classified by genetic mutation, resulting in the development of the targeted agents for each mutant gene. ${ }^{24)}$ Meanwhile, there were 3 cases of improving side effects from these targeted agents by treating with KM; cutaneous adverse reaction of EGFR inhibitors, olmutinib and gefitinib, was improved by herbal medicine therapy. One of the cases reported the improvement of other adverse effects of gefitinib other than skin reaction, such as tingling of extremities, general weakness, and diarrhea, treated with acupuncture and moxibustion. It is reported in the systemic review that complementary herbal medicine therapy to chemotherapy lessens the side effects of it and improves the quality of life, ${ }^{25)}$ and these cases could make the examples.

The types of KM treatment were herbal medicine, acupuncture, electro-acupuncture, moxibustion, pharmacopuncture, cupping, and so on. The most frequently used herbal medicine was allergen-removed Rhus verniciflua Stokes (aRVS). Rhus verniciflua has been used to treat tumors for a long time, and herbal medicine obtained from dried Rhus verniciflua is geonchil (乾漆). ${ }^{26)}$ Geonchil is the representative herb to treat neoplastic diseases, which is described as "old static blood (瘀血) and accumulation (積) resulted from old static blood" in traditional Korean medicine. ${ }^{27)}$ Experimental studies showed that geonchil induces apoptosis, ${ }^{28)}$ inhibits tumor cell growth ${ }^{29)}$ and angiogenesis, ${ }^{30)}$ and the retrospective chart review of 40 cases confirmed that aRVS extends survival in lung cancer patients. ${ }^{31)}$ Following aRVS was the cases of using HAD, HAP, and Soramdan. HAD is anti-cancer herbal medicine consisting of Coicis Semen, Panax notoginseng, Radix Hippocampus Kelloggii, Cordyceps Militaris, Santsigu Tuber, Ginseng Radix, Bovis Calculus, Margarita, and Moschus, with its effect proven by the experiments. ${ }^{32,33)}$ HAP is composed of Coix lachrymal Semen, Panax notoginseng Radix, Hippocampus kelloggi, Cordyceps Militaris, Satsigu Tuber, Ginseng Radix, Bovis Calculus, Margarita, and Moschus. Panax notoginseng and Cordyceps militaris are known to block vascular epithelial growth factor (VEGF) and induce apoptosis to prevent recurrence and metastasis of cancer by experiments. ${ }^{34), 35)}$ Also, the water extract of HAP is reported to inhibit tumor cell growth by anti-angiogenic activity $^{36)}$ and to be safe without blood toxicity through animal testing. ${ }^{37)}$ Soramdan is composed of wild ginseng. ${ }^{90)}$ Previous studies reported that wild ginseng showed anticancer effect in the human lung cancer cell line ${ }^{38)}$ and in patients with lung cancer relatively. ${ }^{39)}$ The cases analyzed in this study are the instances of applying these experimental research in clinics based on effective results - which is significant in that the experimental studies indeed reach out to clinics for clinical verification. More and more case studies need accumulate to build the evidence of cancer treatment. Other herbal medicine to improve the patient's symptoms are Bojung-ikki-tang, Maekmundong-tang, Geumsuyukunjun, and so on. These are prescribed to 
treat typical symptoms of lung cancer patients such as cough, dyspnea, and general weakness. In the actual clinical practice, there are more instances of treating the symptoms accompanied by cancer as the primary purpose of treatment, ${ }^{40)}$ so these prescriptions may be helpful for practitioners. Besides, two cases reported improvement of adverse effect on skin from epithelial growth factor receptor inhibitor by using modified Samul-tang, which can be useful in treating patients with similar side effects of chemotherapy in KM clinics.

Acupuncture points used in acupuncture and moxibustion treatment were varied; the mostly used were su points on bladder meridian, such as BL13 and BL17, and some cases used lung-tonifying acupuncture of Sa-am acupuncture. Other tools of treatment included electroacupuncture, pharmacopuncture, light therapy, aromatherapy, sitz bath, and foot bath, which is considered to satisfy the patients' needs if used in clinics.

Various objective and subjective measurements to evaluate efficacy were suggested in the case reports. The objective measurements included imaging tests to identify the tumor size or pleural effusion, tumor response according to RECIST, blood test to check the level of tumor marker, and the length of survival. The subjective measurements were changes in symptoms of subjective complaints and the QOL. Evaluation on the QOL of cancer patients is considered important in treating them and assessing their status along with the survival rate and the tumor response, ${ }^{41)}$ and there has been plenty of studies on the QOL. ${ }^{42)}$ Although specialized questionnaires by type of cancer have been developed, there were only two cases of using such a questionnaire in this study unfortunately. More objectified evaluation would be possible if practitioners further utilize these questionnaires when assessing the patients' QOL.

Safety evaluation measurement was done by blood test and observing adverse effects. The liver and kidney function was tested in blood test. Adverse effects were analyzed with the international criteria, Common Terminology Criteria for Adverse Events (NCI-CTCAE), only in 8 cases. The use of herbal medicine is increasing followed by the growing interest in the safety of herbal medicine. Under current medical system of South Korea, a number of patients including cancer patients take medicines prescribed in hospitals of KM and Western medicine respectively. These medicines are metabolized by drug-metabolizing enzymes and may effect activation of them. Some of them may turn into toxic substance through metabolic process, so they need special caution when used together. ${ }^{43)}$ Therefore the safety evaluation should be taken strictly, and adverse effects need to be evaluated based on international criteria to accumulate data for more precise and safe KM treatment, not only in research but also in clinics.

On the perspective of effectiveness and safety, these studies had effects in diverse ways and safety as well. Regarding the tumor response, partial response was reported in 12 cases, stable disease was in 22 cases, $50 \%$ of the total cases, which is a high level of tumor response. Furthermore, all 11 cases with the evaluation on the length of survival showed prolonged survival than the expectancy of corresponding stage, with the stable QOL during survival. Although the cases are small in number and limited to lung cancer only, but the results are encouraging. Meanwhile, more and more patients demand improvement of side effects and QOL according to the cases reviewed in this article in $\mathrm{KM}$ practice of cancer. 16 out of 17 cases with QOL evaluation reported improved or stable QOL, and side effects of surgery or chemotherapy were improved in all relevant cases.

We integrated and analyzed case reports on lung cancer in the society of KM in Korea. For practitioners, it is important to check cases and apply them in clinical 
practice, and evidence based medicine (EBM) is considered to be the fundamental knowledge and skill of medical profession when making clinical decisions. ${ }^{17)}$ EBM is defined as integration of the latest research evidence, clinical experience of the practitioner, and the value of patients. ${ }^{44)}$ The latest research evidence means patient-centered clinical study, and case reports are considered low level of evidence to apply in studies. However, under current situation where large-scale randomized controlled trials about KM treatment on lung cancer are scarce, case reports can be alternative to higher level of evidence. Furthermore, the insight into patient, intervention, and outcome drawn from the discussion will make the foundation for well-designed clinical trial suitable for the characteristics of $\mathrm{KM}$ treatment.

\section{References}

1. Schiller JH, Harrington D, Belani CP, Langer C, Sandler A, Krook J, et al. Eastern Cooperative Oncology Group. Comparison of four chemotherapy regimens for advanced non-small-cell lung cancer. $\mathrm{N}$ Engl $\mathrm{J}$ Med. 2002;346(2):92-98.

2. American Cancer Society: Cancer Facts \& Figures 20OED. Atlanta: American Cancer Society; 2017. Available at: URL:https://www.cancer.org/research/ cancer-facts-statistics/all-cancer-facts-figures/ca ncer-facts-figures-2017.html.

3. National Cancer Center. Annual report of cancer statistics in Korea in 2015, Gyeonggi-do:National Cancer Center. 2017 Dec. Available at: URL:http://ncc.re.kr/cancerStatsView.ncc?bbsnu $\mathrm{m}=438 \&$ searchKey=total\&search Value $=\&$ page Num=1.

4. Kim YH, Kim YT, Kim HK, Park IG, Wu HG, Song SY, et al. Lung cancer guideline. Korean Association for Lung Cancer. 2010.
5. Shim JK, Kwon HJ, Jung MK, Oh JM, Park SH, Shin KM, et al. Fatigue and its Related Factors in Lung Cancer Patients on Chemotherapy. The chung-Ang Journal of Nursing 2003;7(1):9-15.

6. Hong M, Shim SH, Yoon SH, Choi JY, Kim YS. Development and Validity Test on Preliminary Critical Pathway of Integrative Medicine for Lung Cancer Patients Treated with Chemotherapy. Journal of Korean Oriental Internal Medicine. 2017;38(6):917-29.

7. Jung HJ, Kim JD. The Role of Korean Medicine for Treatment and Management for Lung Cancer. Journal of Korean Traditional Oncology. 2015:20(1);15-54.

8. Nissen T, Wynn R. The history of the case report: a selective review. J R Soc Med. 2014 Mar 12;5(4):1-5.

9. Nissen T, Wynn R. The clinical case report: a review of its merits and limitations. BMC Res Notes. 2014;7:264.

10. Nissen T, Wynn R. The recent history of the clinical case report: a narrative review. JRSM Short Reports. 2012;3(12):87.

11. Smith R. Why do we need Cases Journal? Cases J. 2008;1(1):1.

12. Vandenbroucke JP. Case reports in an evidence-based world. J R Soc Med. 1999 Apr;92(4):159-163.

13. Arnaiz JA, Carne X, Riba N, Codina C, Ribas J, Trilla A. The use of evidence in pharmacovigilance: case reports as the reference source for drug withdrawals. Eur J Clin Pharmacol. 2001;57(1):89-91.

14. Caban-Martinez A, Garcia-Beltran WF. Advancing medicine one research note at a time: the educational value in clinical case reports. BMC Research Notes. 2012;5:293.

15. Jang SB, et al. A Study on Complementary Alternative Therapy Used by Cancer Patients in 
Korea. Korean J Adult Nurs. 2006;18(1): 92-101.

16. Kim KS, Kim SH, Eo WK, Cheon SH, Eom SK, Jo HJ. Clinical research methodology for Traditional Korean Medicine treatment of Lung cancer: Evidence-based approach. The Journal of Oriental Medical Classics. 2010;23(4):39-62.

17. Lee YM. Introduction to Evidence Based Medicine. Vascular Specialist International. 2003;19(2):212-219.

18. Firat AC, Araz C, Kayhan Z. Case reports: Should we do away with them? J Clin Anesth. 2017 Feb;37:74-76.

19. Soda M, Choi YL, Enomoto M, Takada S, Yamashita Y, Ishikawa S, et al. Identification of the transforming EML4-ALK fusion gene in non-small-cell lung cancer. Nature. 2007;448(7153):561-566.g ZF, Yu DQ. Chemical constituents of the stem bark of Morus cathayana. J Asian Nat Prod Res. 2010;12:505-15.

20. Kim YC, Kwon YS, Oh IJ, Kim KS, Kim SY, Ryu JS, et al. National Survey of Lung Cancer in Korea. J Lung Cancer. 2007;6(2):67-73.

21. Korean Association of Traditional Oncology. Integrative Oncology of Korean Medicine. Seoul:Gunja. 2013:243.

22. Wang W, Zhang X, Qin JJ, Voruganti S, Nag $\mathrm{SA}$, Wang $\mathrm{MH}$, et al. Natural product ginsenoside 25-OCH3-PPD inhibits breast cancer growth and metastasis through down-regulating MDM2. PLoS One. 2012;7(7):e41586.

23. Cheng X, Gu J, Zhang M, Yuan J, Zhao B, Jiang $\mathrm{J}$, et al. Astragaloside IV inhibits migration and invasion in human lung cancer A549 cells via regulating PKC- $\alpha$-ERK1/2-NF- $\kappa$ B pathway. Int Immunopharmacol. 2014 Nov;23(1):304-313.

24. Cancer Genome Atlas Research Network. Comprehensive molecular profiling of lung adenocarcinoma. Nature. 2014;511(7511):543550.
25. Chen S, Flower A, Rirchie A, Liu J, Molassiotis $\mathrm{A}, \mathrm{Yu} \mathrm{H}$, et al. Oral Chineses herbal medicine as an adjuvant treatment during chemotherapy for non-small cell lung cancer: A systematic review. Lung Cancer. 2010:68(2);137-145.

26. United Department of Herbology. Herbology. Seoul:Younglimsa. 1991:439.

27. Kim KS. Case Study: Regression of a Residual Tumor and Prolongation of Overall Survival with Allergen-removed Rhus verniciflua Stokes after Chemoradiotherapy in Locally Advanced Non-small Cell Lung Cancer. The Journal of Korean Oriental Internal Medicine. 2015;36(2):200-206.

28. Kim JH, Kim HP, Jung CH, Hong MH, Hong $\mathrm{MC}$, Lee SD, et al. Inhibition of cell cycle progression via p27Kip1 upregulation and apoptosis induction by an ethanol extract of Rhus verniciflua stokes in AGS gastric cancer cells. Int J Mol Med 2006;18(1):201-208.

29. Jang HS, Kook SH, Son YO, Kim JG, Jeon YM, Jang YS, et al. Flavonoids purified from Rhus verniciflua Stokes actively inhibit cell growth and induce apoptosis in human osteosarcoma cells. Biochim Biophys Acta. 2005;1726(3):309-316.

30. Lee JD, Huh JE, Jeon G, Yang HR, Woo HS, Choi DY, et al. Flavonol-rich RVHxR from Rhus verniciflua Stokes and its major compound fisetin inhibits inflammation-related cytokines and angiogenic factor in rheumatoid arthritic fibroblast-like synovial cells and in vivo models. Int Immunopharmacol. 2009 Mar;9(3):268-276.

31. Cheon SH, Kim KS, Kim S, Jung HS, Choi WC, Eo WK. Efficacy and safety of Rhus verniciflua stokes extracts in patients with previously treated advanced non-small cell lung cancer. Forschende Komplementarmedizin. 2011;18(2):77-83.

32. Lee DE, Lee SY, Kim JS, Cho CK, Yoo HS, Choi SJ. Antitumor effect of HangAm-Dan (HAD) 
and its ingredients on Calu6 and MCF-7 human cancer cell lines. J Korean Oriental Med. 2009;30(5):50-60.

33. Yoo HS, Kim SH, Lee YH, Cho CK, Lee KH. Two cases of endometrial cancer treated with palliative herbal medication Hangam-dan (HAD). J Korean Oriental Med. 2007;28(4):176-180.

34. Park SC. Yoo HS. Park C. Cho CK. Kim GY. Kim W J. et al. Induction of apoptosis in human lung carcinoma cells by the water extract of Panax notoginseng is associated with the activation of caspase-3 through downregulation of Akt. Int $\mathrm{J}$ Oncol. 2009;35(1):121-127.

35. Park SE. Yoo HS. Jin CY. Hong SH. Lee YW. $120 \mathrm{Kim} \mathrm{BW}$. et al. Induction of apoptosis and inhibition of telomerase activity in human lung carcinoma cells by the water extract of Cordyceps militaris. Food Chem Toxicol. 2009;47 (7):1667-1675.

36. Bang JY. Kim EY. Shim TK. Yoo HS. Lee YW. Kim YS. et al. Analysis of anti-angiogenic mechanism of HangAmDan-B (HAD-B). a Korean traditional medicine, using antibody microarray chip. BioChip 1. 2010;4(4):350-355.

37. Lee HJ, Lee G, Y00 HS. Toxicological Study on Hang-Am-Plus in Mice. J Acupunct Meri'dian Stud. 2011;4(1):54-60

38. Yang MC, Seo DS, Choi SU, Park YH, Lee KR. Polyacetylenes from the Roots of Cultivated-Wild Ginseng and Their Cytotoxicity In Vitro. Arch Pharm Res. 2008;31(2):154-159.

39. Lee JH, Kwon KR, Cho CK, Han SS, Yoo HS. Advanced cancer cases treated with cultivated wild ginseng phamacopuncture. J Acupunct Meridian Stud. 2010;3(2):119-124.

40. Seong S. Lung Cancer, the speed is the answer. The Seed Books. 2013:154-155.

41. Lee NH, Cho JH, Son CG, Yoo HS, Lee YW, Yoon DH, et al. Analysis of Studies on Quality of Life according to Cancer Types and Symptoms. Korean J Orient Int Med. 2006: 27(3):555-560.

42. Lee EH, Park HB, Kim MW, Kang SH, Lee HJ, Lee $\mathrm{WH}$, et al. Analyses of the Studies on Cancer-Related Quality of Life Published in Korea. Radiat Oncol J. 2002;29(4):359-366.

43. Seong EJ, Lee MY, Seo CS, Shin HK, Cho JW, $\mathrm{Ha}$ H. Sub-acute toxicity and effect of Hwangryunhaedok-tang on human drug-metabolizing enzymes. J Korean Med. 2017;38(2):15-30.

44. Luckmann R. Evidence-Based Medicine: How to Practice and Teach EBM, 2nd Edition: By David L. Sackett, Sharon E. Straus, W. Scott Richardson, William Rosenberg, and R. Brian Haynes, Churchill Livingstone, 2000. Journal of Intensive Care Medicine. 2000;16(3):155-156.

45. Park BK, Cho CK, Kwon KR, Yoo HS. A Case Report for Stage IIIB Squamous Cell Lung Carcinoma Patient Treated with Cultured Wild Ginseng Pharmacopuncture Therapy. Journal of pharmacopuncture. 2007;10(3):143-147.

46. Lee WC, Shin KS. A Case of Metastatic Brain Tumor Originated from Lung Cancer treated by Oriental Medicine. Journal of Korean Oriental Oncology. 1999;5(1):151-158.

47. Cha ES, Jo IH, Lee GG, Jo YM, Jeong HJ, Jeong SG, et al. A Case of Postobstructive Pneumonia in Lung Cancer Patient by Oriental Medical Treatment. Journal of Korean Oriental Oncology. 1997;3(1):207-219.

48. Park SJ, Kang HJ, Park JH, Cho CK, Yoo HS. A case report of a clinically diagnosed advanced lung cancer patient after treatment with Gunchilgyebok-Jung. Journal of Korean Traditional Oncology. 2015;20(1):23-29.

49. Choi YN, Kim J, Bae K, Cho CK, Yoo HS. A Case of Non-Small Cell Lung Carcinoma Patient Who Improved Skin Rash Due to Olmutinib by Administration of Nobongsangki-Jeong. Journal 
of Korean Traditional Oncology. 2017;22(1):13-22.

50. Lee SH, Lee JS, Jung H, Choi WC, Kim KS. A Case Report of Non-small Cell Lung Cancer with Brain Metastasis Patient Treated with Banhabaekchulchoenma-tang Gagambang. Journal of Korean Traditional Oncology. 2009;14(1):45-52.

51. Park SJ, Kang HJ, Lee YW, Cho CK, Yoo HS. A Case Report of a Stage IV Non-small Cell Lung Cancer Patient Treated with Modified Yieum-jeon Showing Improvement in Malignant Pleural Effusion and other Respiratory Symptoms The Journal of Internal Korean Medicine. 2014. Sep;35(3): 366-372.

52. Ha SJ, Song SY, Park SJ, Jeon HJ, Lee YW, Cho CK, et al. A Case of Patient with Non-Small Cell Lung Carcinoma Treated with Samchilchoongcho-Jung in Conjunction with Crizotinib. Journal of Korean Traditional Oncology. 2018;23(1):23-32.

53. Park JH, Park SJ, Kang HJ, Cho CK, Han KI, Yoo HS. Tumor-reducing effect of SB injection in a non-small cell lung cancer patient: A case report. Journal of Korean Traditional Oncology. 2014;19(1):61-68.

54. Park JH, Cha J, Lee JS, Jung HS, Lee SH, Choi WC, et al. A Case of Non-small Cell Lung Cancer Patient Whose Dermatologic Adverse Reactions Associated with the Epidermal Growth Factor Receptor-inhibitors were Relieved with the Treatment of a Herbal medicine, Samultang-gagambang. Journal of Korean Traditional Oncology. 2011;16(2):55-61.

55. Bang SH, Kwon KR, Yoo HS. Two Cases of Non-Small Cell Lung Cancer Treated with Intravenous Cultivated Wild Ginseng Pharmacopuncture. Journal of pharmacopuncture. 2008;11(2):13-19.

56. Kim K, Choi YS, Joo JC, Moon G. A Case Report for Lung Cancer Patient Showing Remission
Treated with Cultivated Wild Ginseng Pharmacopuncture. Journal of Pharmacopuncture. 2011 Dec;14(4):33-37.

57. Park SR, Lee SM, Choi SH, Jung YH, Lee SK. A Case Study of Small-Cell Lung Cancer with Multiple Brain Metastases Patient who Showed Improvement of Delirium and Performance Status. the Journal of Sasang Constitutional Medicine. 2014;25(5):400-408.

58. Choi BL, Song KC, An JJ, Lim SM, Kim MH, Lee JY, et al. A case study of small cell lung cancer patient. Journal of Oriental Chronic Disease. 2003;9(1):85-91.

59. Hong M, JH Lee, Park HL, Lee HY, Cho MK, Han CW, et al. Acute Analgesic Effect of Electroacupuncture on a Cancer Pain in a Small Cell Lung Cancer Patient : a Case Report. Korean Journal of Oriental Physiology \& Pathology. 2014;28(6):689-694.

60. Kim MK, Lee JS, Lee SH, Jung HS, Choi WC, Kim KS. A Clinical Case of Patient with Cancer Pain Treated by Moxibustion. Journal of Korean Traditional Oncology. 2009;14(1):53-59.

61. Song AN, Ahn JH, Kim KS, Jung HS, Lee SK, Choi WC. Long Term Survival of a Patient with Advanced Non-Small Cell Lung Cancer Treated with Allergen Removed Rhus verniciflua Stokes Extract; a Case Report. Journal of Korean Traditional Oncology. 2012;17(1):39-43.

62. Kwon EM, Jerng UM, Kim KS, Lee SK, Choi WC, Yoon SW. Two Cases of Non-small Cell Lung Cancer Patients Treated with Allergen Removed Rhus Verniciflua Stokes Based Traditional Korean Medicine and Chemotherapy. Journal of Korean Traditional Oncology. 2009;14(1):13-20.

63. Yu SM, Eo WK, Yoon SW. One Case Report of Non-small Cell Lung Cancer Patients Treated with Allergen Removed Rhus Verniciflua 
Stokes(aRVS). Journal of Korean Traditional Oncology. 2008;13(1):63-69.

64. Choi SH, Song AN, An JH, Kim EH, Park SJ, Lee SK. A Case Report of a Patient with Advanced Non-Small Cell Lung Cancer Treated with Allergen Removed Rhus Verniciflua Stokes (aRVS). Journal of Korean Traditional Oncology. 2012;17(1):45-53.

65. Park JH, An JH, Lee JS, Jung YH, Lee SH, Cheon $\mathrm{SH}$. A case of the elderly advanced Non-small Cell Lung Cancer Patient Treated with Allergen Removed Rhus Verniciflua Stokes extract. Journal of Korean Traditional Oncology. 2010;15(1);71-77.

66. Kim EH, Park SJ, Choi WC, Lee Sk. A Case Report of Squamous Cell Lung Cancer Patient Treated with Allergen Removed Rhus Verniciflua Stokes Extract. Journal of Korean Traditional Oncology. 2011;16(2):35-41.

67. Kang JY, Kim JY, Son CG, Cho JH. Long-Term Survival of Patients with Lung Cancer Treated by Traditional Korean Medicine Combined with Western Treatment: Report of Two Cases. The Journal of Korean Oriental Internal Medicine. 2015;36(1):58-68.

68. Park SC, Kim DH, Han CW, Park SH, Lee I, Choi JY. Integrated Medicine Therapy for an End-stage Lung Cancer Patient with Brain Metastasis. Journal of Korean Traditional Oncology. 2011;16(1):43-51.

69. Kim JH, Bae KR, Park SJ, Cho CK, Yoo HS. A Case of Patient with Recurring Non-Small Cell Lung Carcinoma Treated with Samchilchoongcho-Jung in Conjunction with Afatinib. The Journal of Korean Oriental Internal Medicine. 2017;38(1):72-80.

70. Park JS, Rhee SH, Yim YN, Jung KY, Jun CY, Park $\mathrm{CH}$, et al. A Clinical Report of the Patient with Limited-Stage Small Cell Lung Cancer. The Journal of Korean Oriental Internal Medicine.
2005;26(3):677-684.

71. Zheng HM, Yoon JW, Lee YW, Cho CK, Oh DS, Yoo HS. Case Series of Advanced Non-Small Cell Lung Cancer Patients Treated with Hang-Am Plus. Journal of Korean Oriental Internal Medicine. 2011;32(1):113-120.

72. Choi KH, Bang JH, Kim JH, Jung SK, Jung HJ. A Clinical Case Report of a Lung Cancer Patient with Shoulder Pain after Lobectomy. Journal of Korean Traditional Oncology. 2010;15(2):79-87.

73. Park JW, Jun HJ, Cho CK, Lee YW. One Case Study of a Non Small Cell Lung Cancer Patient Experiencing Gefitinib Adverse Effects Managed by Traditional Korean Medicine. Journal of Korean Traditional Oncology. 2012;17(1):9-16.

74. Gu JH, Kim SR, Im EY, Kim SJ, Kim JD. One Clinical Case Report of Lung Cancer Patient with Depression and Anxiety Disorder Improved by Korean Traditional Medical Treatment and Breathing Meditation. Korean Journal of Oriental Physiology \& Pathology. 2011;25(6):1102-1107.

75. Choi JH, Yoo HS, Son CG, Cho CK. Regression of small cell lung carcinoma by herbal medicine:a case with an 8-year follow-up. Journal of Korean Traditional Oncology. 2005;10(1):87-91.

76. Yoo SH, Yoo HS, Cho CK, Lee YW. A Case Report for Recurred Squamous Cell Lung Carcinoma Treated with Hang-Am-Dan : 7-year follow-up. Journal of Korean Oriental Internal Medicine. 2007;28(2):385-390.

77. Park SR, Lee SM, Lee SK. A Case of the Advanced Non-Small Cell Lung Cancer Patient Who Showed Symptoms Improvement. Journal of Sasang Constitutional Medicine. 2015;27(3):326-332.

78. Kim KS, Jung TY, Yoo HS, Lee YW, Cho CK. Case Series of Advanced Non-small Cell Lung Cancer Patients Treated with Hang-Am-Plus. Journal of Korean Oriental Internal Medicine. 2009;30(4):893-900. 
79. Lee JS, Park JH, Chae J, Kim KS, Jung HS, Lee $\mathrm{SH}$, et al. Case Report : Long-term Survival and Good-Quality of Life in Metastatic Non-small Cell Lung Cancer Patients Treated with Allergen-removed Rhus verniciflua Stokes (aRVS) as Sequential and Concurrent Treatment with Chemotherapy. Journal of Korean Oriental Internal Medicine. 2011;32(1):129-135.

80. Son JY, Choi GH, Yoo HJ, Kim HJ, Goo JW, Park MY, et al. One Clinical Case Report of Lung Cancer Patient with Dyspnea Prescribed Oriental Medicine. Journal of Korean Oriental Internal Medicine. 2009;30(1):233-240.

81. Yi JE, Sun SJ, Jung YS, Kim BW. A Case Report on the Improvement of Lung Cancer Patient's Symptoms by Keumsooyukgun-geun. J of Oriental Chr Dis. 2003;9(1):70-77.

82. Park HM, Kim SY, Jung IC, Lee YW, Cho CK, Yoo HS. Integrative tumor board: case report and discussion from East-West Cancer Center. Integr Cancer Ther. 2010;9(2):236-245.

83. Lee SH, Kim KS, Choi WC, Yoon SW. The Concurrent Use of Rhus verniciflua Stokes as Complementary Therapy with Second or More Line Regimens on Advanced Non-small-cell Lung Cancer: Case Series. The Journal of Korean Oriental Medicine. 2009;30(6):112-117.

84. Hong MN, Park HL, Choi JH, Choi JY, Bae GE, Han CW, et al. A Case Report of a Mucinous Adenocarcinoma Patient Treated with Korean Medicine after Wedge Resection of the Lung. J. Int. Korean Med. 2016;37(5):776-785.

85. Song SY, Jeon $\mathrm{H}$, Lee S. Improvement of Pneumonia in a Patient with Non-Small Cell Lung Cancer Treated with Herbal Medicine after Cessation of Antibiotics - a Case Report. J Korean Med. 2017;38(2):78-84.

86. Lee SH, Kim KS, Choi WC, Yoon SW. Successful Outcome of Advanced Pulmonary Adenocarcinoma
With Malignant Pleural Effusion by the Standardized Rhus Verniciflua Stokes Extract: A Case Study. EXPLORE. 2009;5(4):242-244.

87. Kim K, Lee S. Remission of Unresectable Lung Metastases from Rectal Cancer After Herbal Medicine Treatment: A Case Report. EXPLORE. 2016;12(4):259-262.

88. Jang HJ, Lee SA, Sin S, Kim S, Han G. Combined Treatment for Lung Metastasis from Hepatocellular Carcinoma: A Case Report. EXPLORE. 2018;14(5):375-388.

89. Lim DH, Park KW, Lee SI. Spontaneous Complete Regression of Multiple Metastases of Hepatocellular Carcinoma: A Case Report. Oncology Letters. 2014;7(4):1225-1228.

90. Lee DH, Kim SS, Shin S, Woo CR, Han JB. A Case of Metastatic Bladder Cancer in Both Lungs Treated with Korean Medicine Therapy Alone. Case Rep Oncol. 2014;7(2):534-540.

91. Lee DH, Shin S, Kim SS, Han JB. A Case of Stage IV Non-Small Cell Lung Cancer Treated with Korean Medicine Therapy Alone. Case Rep Oncol. 2013;6(3):574-578.

\section{ORCID}

Gajin Han: $\quad$ https://orcid.org/0000-0003-2106-652X Haegue Shin: https://orcid.org/0000-0002-3297-8164 Sin Seong: https://orcid.org/0000-0003-0081-439X Sungsu Kim: https://orcid.org/0000-0003-4486-599X 